\title{
Variability of Electrocardiographic Imaging Within and Between Leadsets
}

\author{
Job Stoks ${ }^{1,2,3}$, Bianca D van Rees ${ }^{1}$, Sanne A Groeneveld ${ }^{4}$, Diantha JM Schipaanboord ${ }^{4}$, Lennart \\ Blom $^{4}$, Rutger J Hassink ${ }^{4}$, Matthijs JM Cluitmans ${ }^{1}$, Ralf Peeters ${ }^{2}$, Paul GA Volders ${ }^{1}$ \\ ${ }^{1}$ Department of Cardiology, Maastricht University Medical Centre, Maastricht, the Netherlands \\ ${ }^{2}$ Data Science and Knowledge Engineering, Maastricht University, Maastricht, the Netherlands \\ ${ }^{3}$ BIOMED, Hasselt University, Hasselt, Belgium \\ ${ }^{4}$ Department of Cardiology, University Medical Centre, Utrecht, the Netherlands
}

\begin{abstract}
The variability of the inverse solution provided by electrocardiographic imaging (ECGI) is largely unknown when comparing different leadsets or (similar) beats. In four patients, we compared activation times (ATS), recovery times (RTs), and correlation coefficients during QRS complex and STT segment between: 1) consecutive sinus beats within one leadset, and 2) multiple beats for two leadsets. Furthermore, reasons behind differences in $R T$ were investigated. Zero-th order Tikhonov regularization was used to reconstruct ventricular epicardial potentials. A spatiotemporal estimation method was then used to determine the ATs and RTs from the reconstructed epicardial electrograms. Inter-leadset differences were generally low for ATs, but exceeded intraleadset beat-to-beat variations. RTs, however, showed larger variation independent of leadset. Differences in RTs between beats or leadsets could partially be explained by low T-wave amplitudes and high levels of noise, which suggests that $R T$ determination may require more advanced methods in these cases. These findings increase our understanding of the consequences of electrode placement for the inverse solution, as well as our understanding of the complexities of recovery time estimation in ECGI.
\end{abstract}

\section{Introduction}

Electrocardiographic imaging (ECGI) is a modality that noninvasively images electrical activation and recovery on the heart surface based on the combination of body-surface EG mapping and a patient-specific torso-heart geometry. For various aspects, ECGI provides more detailed information than the clinical electrocardiogram (ECG). It has previously been validated by our group [1] and others.

However, the stability of the inverse solution provided by ECGI remains largely unknown. For example, this holds true for inverse reconstruction when using reducedleadsets and when studying stable beat-to-beat characteristics. To improve assessment, we compared epicardial potential maps and isochrones for different beats and for two different leadsets. In-vivo recordings from four patients were used. Quantitative differences between inverse solutions and the reasons behind these differences are evaluated.

\section{Methods}

\subsection{BSPM and geometry}

The study was approved by the medical ethical committee of Maastricht University Medical Center. All patients gave written informed consent before entering the study. A CT scan was performed with intravenous iodine contrast medium and the diastolic phase of the torso-heart geometry was reconstructed. Subsequently, segmentation of the ventricular epicardium was performed manually. Geometries were reduced to a median of 2058 (2010-2239) nodes. Prior to the CT scan, two body-surface potential maps (BSPMs) were simultaneously recorded using two independent leadsets at the same time: a 184-channel leadset and a 64-channel leadset (see Figure 1). Both were recorded with an acquisition system of the same manufacturer (BioSemi, Amsterdam, the Netherlands), with a 2048-Hz sampling rate. BSPMs of inter-leadset comparisons were manually checked, to assess whether the input data agreed qualitatively.

\subsection{Preprocessing and reconstruction}

Baseline drift and $50 \mathrm{~Hz}$ noise were removed from the BSPM and the STT-segment was filtered with a $2^{\text {nd }}$ order $40 \mathrm{~Hz}$ lowpass Butterworth filter. Subsequently, inverse reconstructions of epicardial potentials were performed on the basis of an potential-based formulation of ECGI; we used an epicardium-only formulation [1] with zero-th order Tikhonov regularization. Torso models were based on the electrodes of the corresponding leadset. A spatiotemporal estimation method was then used to determine the activation and recovery times from the reconstructed 
epicardial electrograms. [2] This method takes advantage of the spatial relationship between neighboring nodes and their potentials, providing a more accurate inverse solution. [1] Activation times (ATs) and recovery times (RTs) are expressed relative to the first moment of epicardial activation.

\subsection{Comparison of inverse solutions}

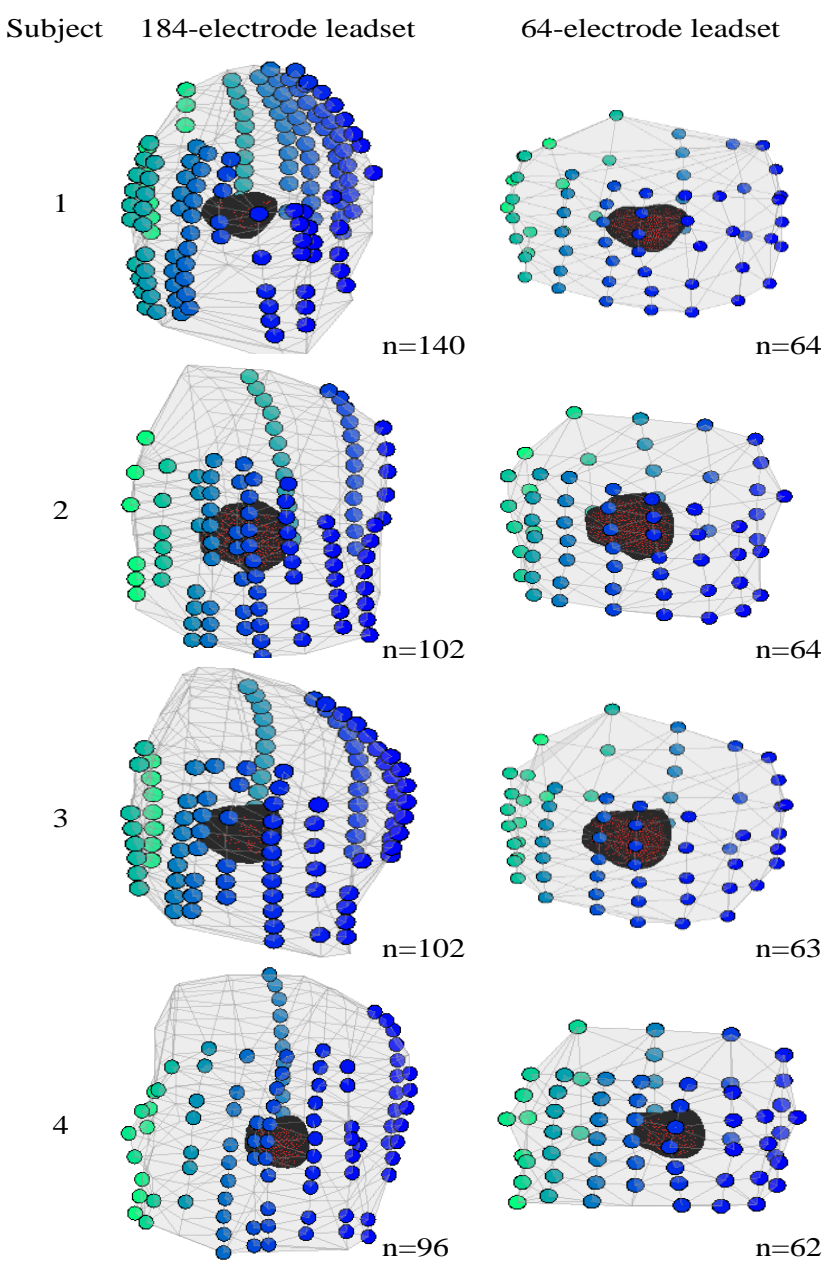

Figure 1: Distributions of electrodes for each patient and each leadset. Electrodes are shown only if they had sufficient signal quality for more than half of the beats, for each subject. Electrodes are colored from left to right. The number of electrodes for each setup is noted as $n$.

To compare the inverse solution of different beats and systems, electrograms of different beats and systems were aligned through the $\mathrm{R}$ peak of the root-mean-square of the BSPM signal, after which all beats were cropped to the same length. Subsequently, several comparisons of the inverse solution were made: 1) consecutive beats with a similar RR interval were compared to each other within one leadset, and 2) single beats were compared between both leadsets. These comparisons were made for sinus beats, ventricular extrasystoles and sinus beats after ventricular extrasystoles. Comparisons were made quantitatively on a node-by-node basis on the epicardium. Nodes at the artificially-capped ventricular base were not taken into account. Results are expressed as absolute differences in AT, RT, and correlation coefficients of electrograms during the QRS complex and STT segment $\left(\mathrm{CC}_{\mathrm{QRS}}\right.$ and $\mathrm{CC}_{\mathrm{STT}}$, respectively). Potential causes for differences were investigated; by addressing $\mathrm{T}$-wave amplitude and noise level at the epicardium. To calculate T-wave amplitude and noise level for each node, each electrogram was first normalized to the highest $R$ peak of the same beat. T-wave amplitude was subsequently calculated as the height of the peak with the largest absolute voltage within the STT segment. Noise level was calculated for each node, as the average squared amplitude of the signal between the end of the QRS complex and the start of the T-wave, divided by the absolute height of the corresponding

R-peak.

\section{Results}

\subsection{Beat-to-beat variability}

For both leadsets, beat-to-beat variability was addressed for consecutive sinus beats. The body-surface electrodes for each leadset and each patient are shown in Figure 1, showing that we varied the number of electrodes in the

Table 1: absolute beat-to-beat AT and RT differences, $\mathrm{CC}_{\mathrm{QRS}}$ and $\mathrm{CC}_{\text {STT }}$ within both leadsets, for each subject. Bottom row shows average results for the 64-leadset. S: subject. Diff: difference. 184-L: 184-leadset. Values shown as median (Q1-Q3).

\begin{tabular}{|c|c|c|c|c|}
\hline & $\begin{array}{l}\text { Absolute } \\
\text { AT diff. } \\
\text { (ms) }\end{array}$ & $\begin{array}{l}\text { Absolute } \\
\text { RT diff. } \\
\text { (ms) }\end{array}$ & $\mathrm{CC}_{\mathrm{QRS}}$ & $\mathrm{CC}_{\text {STT }}$ \\
\hline $\begin{array}{l}\text { S1 } \\
184-\mathrm{L}\end{array}$ & $\begin{array}{l}1.1 \\
(0.5-1.9)\end{array}$ & $\begin{array}{l}12.3 \\
(5.1- \\
26.7)\end{array}$ & $\begin{array}{l}1 \\
(0.99- \\
1.00)\end{array}$ & $\begin{array}{l}0.94 \\
(0.79- \\
0.98)\end{array}$ \\
\hline $\begin{array}{l}\text { S2 } \\
184-\mathrm{L}\end{array}$ & $\begin{array}{l}1.5 \\
(0.6-3.4)\end{array}$ & $\begin{array}{l}11.2 \\
(4.5- \\
22.7) \\
\end{array}$ & $\begin{array}{l}1.00 \\
(0.99- \\
1.00) \\
\end{array}$ & $\begin{array}{l}0.96 \\
(0.89- \\
0.98) \\
\end{array}$ \\
\hline $\begin{array}{l}\text { S3 } \\
184-L\end{array}$ & $\begin{array}{l}1.9 \\
(0.7-3.5)\end{array}$ & $\begin{array}{l}10.9 \\
(4.2- \\
24.3) \\
\end{array}$ & $\begin{array}{l}0.99 \\
(0.98- \\
1.00)\end{array}$ & $\begin{array}{l}0.93 \\
(0.62- \\
0.99)\end{array}$ \\
\hline $\begin{array}{l}\text { S4 } \\
184-\mathrm{L}\end{array}$ & $\begin{array}{l}1.6 \\
(0.6-4.2)\end{array}$ & $\begin{array}{l}12.9 \\
(5.4- \\
27.4) \\
\end{array}$ & $\begin{array}{l}0.97 \\
(0.71- \\
0.99) \\
\end{array}$ & $\begin{array}{l}0.88 \\
(0.53- \\
0.97) \\
\end{array}$ \\
\hline $\begin{array}{l}\text { Avg. } \\
64-L\end{array}$ & $\begin{array}{l}1.4 \\
(0.5-2.5)\end{array}$ & $\begin{array}{l}10.1 \\
(4.2- \\
21.7)\end{array}$ & $\begin{array}{l}0.99 \\
(0.97- \\
1.00)\end{array}$ & $\begin{array}{l}0.94 \\
(0.78- \\
0.98)\end{array}$ \\
\hline
\end{tabular}


184-setup from 96 to 140 throughout the measurements, shifting from homogeneous coverage to a primary anterior coverage.

In total, 28 pairs of consecutive sinus beats were analyzed for each leadset. Results for AT differences, RT differences, $\mathrm{CC}_{\mathrm{QRS}}$ and $\mathrm{CC}_{\mathrm{STT}}$ on a node-by-node basis on the epicardium are shown in Table 1. Results for the 64leadset are averaged only in this table, since these were very similar. Table 1 indicates that results were predominantly similar over different subjects, with a median $\mathrm{CC}_{\mathrm{QRS}}$ and $\mathrm{CC}_{\mathrm{STT}}$ close to 1 , and median $\mathrm{AT}$ differences close to $1 \mathrm{~ms}$. RT differences were much higher than AT differences. Moreover, distributions of $\mathrm{CC}_{\mathrm{STT}}$ and RT differences were skewed with a considerably low first and third quartile, respectively. This indicates that most inverse solutions contained fairly large regional RT differences.

\subsection{Inter-leadset variability}

Qualitatively, BSPMs of both leadsets agreed well (data not shown). Inverse solutions were compared between both leadsets. In total, 83 beats were analyzed, of which 73 sinus beats, 6 ventricular extrasystoles and 4 sinus beats after ventricular extrasystoles. Statistics of differences between leadsets are shown in Table 2 and show good overall agreement, but with skewed distributions. The third quartile of AT and RT differences and the first quartile of correlation coefficients show that regional differences between both leadsets within one beat can be considerably large. RT differences as a function of T-wave amplitude and noise level are shown in Figure 2, showing that both factors contribute to increased RT differences.

\section{Discussion}

In this study, we quantitatively assessed differences in inverse solution between 1) consecutive sinus beats within one leadset, and 2) beats from different origins, between the two leadsets. Comparisons were made on a node-bynode basis on the epicardium.

Median differences between leadsets were relatively small, indicating good overall agreement. However, larger differences between two leadsets occurred regionally. Consequently, clinical interpretation of the inverse solutions of two different leadsets could vary locally. However, these differences still occur (although to a slightly lesser degree) within one leadset. Differences between two leadsets were larger than beat-to-beat differences within a leadset, for all outcome measures except RT which always showed large variation.

Importantly, we showed that RT differences between leadsets can partially be explained by low- amplitude T-waves and high noise levels. This extends our previous work [3] showing that accurately pinpointing the exact RT in an electrogram proves challenging in case of noisy or flat T-waves. Different leadsets (with different numbers of electrodes) may result in differences in $\mathrm{T}$-wave amplitude or noise. Even though the spatiotemporal method [2] was used to define local RT, flat T-waves still proved to be an issue, possibly because ECGI may be prone to project biphasic T-waves as flat, e.g. as visible in figure 2 of ref. [4]. More advanced analysis methods for RT determination should be developed for these areas, possibly considering the amplitude or area of the T-wave, which can also be accurate measures of local RT [5].

Table 2: absolute AT and RT differences, $\mathrm{CC}_{\mathrm{QRS}}$ and $\mathrm{CC}_{\text {STT }}$ between different leadsets, for each subject. S: subject. Diff: difference. Values shown as median (Q1Q3).

\begin{tabular}{|l|l|l|l|l|}
\hline & $\begin{array}{l}\text { Absolute } \\
\text { AT diff. } \\
(\mathrm{ms})\end{array}$ & $\begin{array}{l}\text { Absolute } \\
\text { RT diff. } \\
(\mathrm{ms})\end{array}$ & CC $_{\mathrm{QRS}}$ & CC $_{\text {STT }}$ \\
\hline S1 & $\begin{array}{l}3.6 \\
(1.6- \\
10.4)\end{array}$ & $\begin{array}{l}16.9 \\
(6.4- \\
37.9)\end{array}$ & $\begin{array}{l}0.87 \\
(0.30-0.97)\end{array}$ & $\begin{array}{l}0.84 \\
(0.44-0.97)\end{array}$ \\
\hline S2 & $\begin{array}{l}5.2 \\
(2.3-9.1)\end{array}$ & $\begin{array}{l}10.3 \\
(4.5- \\
21.8)\end{array}$ & $\begin{array}{l}0.93 \\
(0.82-0.98)\end{array}$ & $\begin{array}{l}0.96 \\
(0.85-0.99)\end{array}$ \\
\hline S3 & $\begin{array}{l}4.5 \\
(2.1-8.9)\end{array}$ & $\begin{array}{l}14.2 \\
(5.8- \\
30.6)\end{array}$ & $\begin{array}{l}0.91 \\
(0.72-0.97)\end{array}$ & $\begin{array}{l}0.87 \\
(0.43-0.97)\end{array}$ \\
\hline S4 & $\begin{array}{l}2.1 \\
(0.8-5.5)\end{array}$ & $\begin{array}{l}9.3 \\
(3.2-\end{array}$ & $\begin{array}{l}0.97 \\
(0.71-0.99)\end{array}$ & $\begin{array}{l}0.91 \\
(0.74-0.97)\end{array}$ \\
\hline
\end{tabular}

Importantly, the amount and distribution of electrodes in the 184-leadset differed considerably and only four patients were analyzed. Consequently, this study does not provide a final answer to the comparison between the two leadsets, but rather is a first gross quantification of differences, while the factors that contribute to the observed differences between both leadsets need to be further elucidated.

As previously shown [1], the potential-based formulation of ECGI is subject to possible spatial displacement of electrograms on the cardiac surface. This spatial displacement is currently not predictable, and to the best of our knowledge it cannot be corrected for. It may also be different between beats and leadsets. In this study, comparisons were made on a node-by-node-basis, not taking a possible displacement into account. A more advanced analysis method, which takes the possible spatial displacement into account might be more suitable and may render more positive results. Such spatial displacement may not be critical for specific clinical applications (e.g., 
detection of repolarization abnormalities may be more important than their exact localization).

Our results are in accordance with an earlier study by Cluitmans et al. [6], in which the remaining 59 to 80 electrodes out of 169 produced similar median correlation coefficients to the ground truth as a full setup using 168 electrodes, although the first quartile of differences was considerably lower.

The goal of the current study was to investigate variability and to understand differences, which could ultimately benefit interpretation of inverse solutions and the development of reduced-leadsets. Some specific reduced-leadsets have previously proven to be of sufficient accuracy and quality, especially when the optimal electrode placement was produced by an iterative

\section{A)}

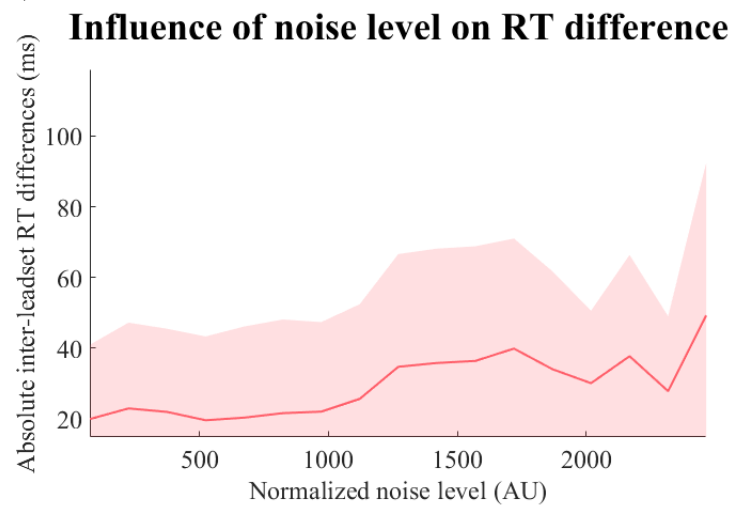

\section{B)}

Influence of $\mathrm{T}$ wave amplitude on $\mathrm{RT}$ difference

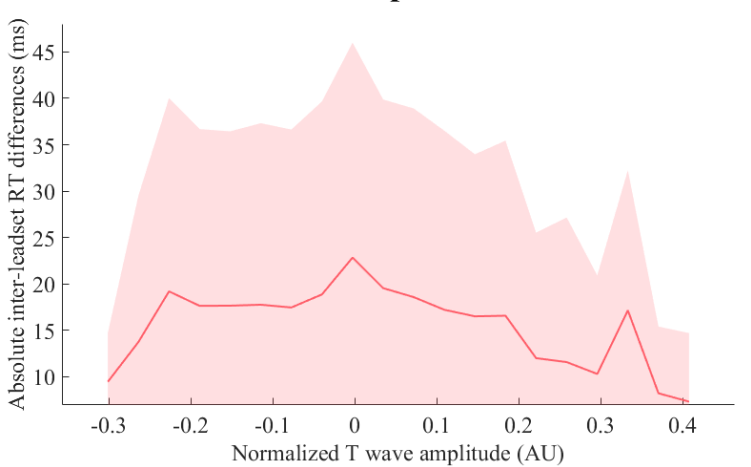

Figure 2: Influence of noise and T-wave amplitude (calculated on the epicardium) on RT difference. Red line shows mean, pink area shows standard deviation. A) an increased level of noise accommodates an increased RT difference. B) a T-wave amplitude approaching 0 contributes to a larger RT difference.

algorithm [7]. In-vivo validation of such an optimal reduced-leadset, incorporating both activation and recovery, may significantly reduce procedural time and the potential of ECGI to be incorporated in clinical practice.

\section{Conclusion}

Inter-leadset differences were generally low even though they exceeded beat-to-beat variations, especially on a regional level. This does, however, not hold for interleadset RTs that were comparable to beat-to-beat variations in recovery times. Differences in recovery times between beats or leadsets could partially be explained through low T-wave amplitudes and high levels of noise, which suggests that RT determination may require more advanced methods in these cases. These findings increase our understanding of the consequences of electrode placement on the inverse solution, as well as our understanding of the intricacies of recovery time estimation in ECGI. We did not investigate spatial displacement of reconstructed electrograms, which may have limited clinical consequences but has large impact on our node-by-node comparisons. As such, this study has identified a lower-bounds on the quality of reconstruction between systems, and has determined that causes of that lower limit lie in noisy and low-amplitude signals.

\section{Disclosure of conflicts of interest}

Matthijs Cluitmans is part-time employed by Philips Research.

\section{References}

[1] M. J. M. Cluitmans et al., "In vivo validation of electrocardiographic imaging," JACC Clin. Electrophysiol., vol. 3, no. 3, pp. 232-242, 2017.

[2] B. Erem, D. H. Brooks, P. M. Van Dam, J. G. Stinstra, and R. S. MacLeod, "Spatiotemporal estimation of activation times of fractionated ECGs on complex heart surfaces," Proc. Annu. Int. Conf. IEEE Eng. Med. Biol. Soc. EMBS, pp. 5884-5887, 2011.

[3] J. Stoks, M. J. M. Cluitmans, R. Peeters, and P. G. A. Volders, "The influence of using a static diastolic geometry in ECG imaging," Comput. Cardiol. (2010)., vol. 2019-Septe, pp. 2-5, 2019.

[4] L. R. Bear et al., "Advantages and pitfalls of noninvasive electrocardiographic imaging," $J$. Electrocardiol., vol. 57, pp. S15-S20, 2019.

[5] M. Potse, A. Vinet, T. Opthof, and R. Coronel, "Validation of a simple model for the morphology of the T wave in unipolar electrograms," Am. J. Physiol. Hear. Circ. Physiol., vol. 297, no. 2, pp. 1-4, 2009.

[6] M. J. M. Cluitmans et al., "In-vivo evaluation of reduced-lead-systems in noninvasive reconstruction and localization of cardiac electrical activity," Comput. Cardiol. (2010)., vol. 42, pp. 221-224, 2015.

[7] F. G. No, Y. S. Dogrusoz, O. N. Onak, and G.-W. Weber, "Reduced leadset selection and performance evaluation in the inverse problem of electrocardiography for reconstructing the ventricularly paced electrograms," J. Electrocardiol., 2020. 